\title{
Association of Sarcopenia and Body Composition With Short-term Outcomes After Liver Resection for Malignant Tumors
}

\author{
Giammauro Berardi, MD, PhD; Giulio Antonelli, MD; Marco Colasanti, MD, PhD; Roberto Meniconi, MD; Nicola Guglielmo, MD; \\ Andrea Laurenzi, MD; Stefano Ferretti, MD, PhD; Giovanni Battista Levi Sandri, MD, PhD; Alessandra Spagnoli, PhD; \\ Giovanni Moschetta, MD; Vincenzo Schininà, MD; Mario Antonini, MD; Massimo Marignani, MD; Giuseppe Maria Ettorre, MD
}

IMPORTANCE Previous retrospective studies have shown that sarcopenia substantially alters the postoperative and oncological outcomes after liver resection for malignant tumors. However, the evidence is limited to small retrospective studies with heterogeneous results and the lack of standardized measurements of sarcopenia.

OBJECTIVE To investigate the role of sarcopenia as a risk factor associated with 90-day morbidity after liver resection for malignant tumors.

DESIGN, SETTING, AND PARTICIPANTS This cohort study included 234 consecutive patients undergoing liver resection for malignant tumors at San Camillo Forlanini Hospital, Rome, Italy, between June 1, 2018, and December 15, 2019. Muscle mass and strength were assessed using the skeletal muscle index (SMI) on preoperative computed tomographic scans and the handgrip strength test, respectively. Patients were then divided into the following 4 groups: group A (normal muscle mass and strength), group B (reduced muscle strength), group C (reduced muscle mass), and group D (reduced muscle mass and strength).

MAIN OUTCOMES AND MEASURES The primary outcome of the study was 90-day morbidity. The following secondary outcomes were investigated: 90-day mortality, hospital stay, and readmission rate.

RESULTS Sixty-four major and 170 minor hepatectomies were performed in 234 patients (median age, 66.50 [interquartile range, 58.00-74.25] years; 158 men [67.5\%]).

The median SMI of the entire population was 46.22 (interquartile range, 38.60-58.20) $\mathrm{cm} / \mathrm{m}^{2}$. The median handgrip strength was 30.80 (interquartile range, 22.30-36.90) kg. Patients in group $D$ had a statistically significantly higher rate of 90 -day morbidity than patients in the other groups ( $51.5 \%$ [35 of 68 ] vs $38.7 \%$ [ 29 of 75$]$ in group $C_{1} 23.1 \%$ [3 of 13 ] in group $B$, and $6.4 \%$ [5 of 78 ] in group $A_{;} P<.001$ ). Compared with patients in the other groups, those in group $D$ had a longer hospital stay (10 days vs 8 days in group $C, 9$ days in group $B$, and 6 days in group $A ; P<.001)$, and more patients in this group were readmitted to the hospital (8.8\% [6 of 68 ] vs 5.3\% [4 of 75] in group C, 7.7\% [1 of 13 ] in group $B$, and $0 \%$ [O of 78] in group $A_{;} P=.02$ ). Sarcopenia, portal hypertension, liver cirrhosis, and biliary reconstruction were independent risk factors associated with 90-day morbidity.

CONCLUSIONS AND RELEVANCE Sarcopenia appears to be associated with adverse outcomes after liver resection for malignant tumors. Both muscle mass measurements on computed tomographic scans and muscle strength assessments with the handgrip strength test should be performed at the first clinical encounter to better classify patients and to minimize the risk of morbidity.

Author Affiliations: Author affiliations are listed at the end of this article.

Corresponding Author: Giammauro Berardi, MD, PhD, Department of General Surgery and Liver Transplantation, San Camillo Forlanini Hospital, Via Giacomo Folchi 6a, 00152 Rome, Italy (gberardi1@gmail.com). 
S urgical treatment with negative margins is the foundation for most gastrointestinal malignant tumors because it offers long-term survival compared with locoregional or systemic therapies. ${ }^{1-3}$ In addition, abdominal surgery is associated with both postoperative mortality and morbidity depending on the condition of the patient and the type of intervention. Despite substantial improvements in perioperative management and surgical techniques, hepatobiliary surgery carries a high risk of morbidity and mortality, reaching rates of $20 \%$ to $50 \%$ as reported in the literature. ${ }^{4-6}$ Liver resections are considered major surgical procedures, frequently involving a large incision, extensive mobilization, long operative time, bleeding risk, and a delicate postoperative course. Furthermore, patients with malignant tumors often have impaired liver function because of cirrhosis, neoadjuvant chemotherapy, and cholestasis, increasing the chance of unexpected postoperative events. ${ }^{7,8}$ Therefore, it is necessary to accurately select patients scheduled for liver surgery to provide the best oncological treatment while minimizing the risk of morbidity.

Age, performance status, comorbidities, and body mass index (BMI) are well-known patient-related variables that substantially alter morbidity rates after liver surgery; however, variables with the potential to better estimate short-term outcomes after major hepatobiliary surgery have been described. ${ }^{9,10}$ Sarcopenia is the degenerative loss of muscle mass, strength, and function, and its prevalence among patients undergoing surgery for gastrointestinal cancer ranges between $30 \%$ and $74 \% .{ }^{11}$ It has been associated with worse short-term and long-term outcomes after liver transplantation and after surgery across a wide range of cancers, such as colorectal, gastric, esophageal, pancreatic, and liver. ${ }^{11,12}$ It has been suggested that the presence of sarcopenia may better predict outcomes of liver surgery compared with the American Society of Anesthesiologists score, performance status, and BMI. ${ }^{13-19}$ Previous studies ${ }^{10,13,15,20}$ have highlighted that impaired muscle mass substantially alters the postoperative and oncological outcomes after surgery for hepatocellular carcinoma and colorectal liver metastases.

Although it has been previously reported that sarcopenia substantially alters oncological outcomes after liver resection for malignant tumors, the available small studies ${ }^{11,13}$ are limited by heterogeneity of results, a retrospective design, and the lack of standardized measurements of sarcopenia, thus restricting applicability in current clinical practice. Because of a lack of high-quality evidence, the objective of this prospective cohort study was to investigate the role of sarcopenia as a risk factor associated with outcomes after liver resection for malignant tumors.

\section{Methods}

Between June 1, 2018, and December 15, 2019, all consecutive patients undergoing liver resection for malignant tumors at San Camillo Forlanini Hospital, Rome, Italy, were prospectively enrolled in this study. Patient demographics, disease presentation, type of surgical approach and type of resection per-

\section{Key Points}

Question Does sarcopenia alter the postoperative outcomes of patients undergoing liver resection for malignant tumors?

Findings In this cohort study of 234 patients, sarcopenia was associated with a higher rate of 90-day morbidity, a longer hospital stay, and a higher readmission rate. Furthermore, sarcopenia was an independent risk factor associated with 90-day morbidity.

Meaning Muscle mass and strength should be assessed before liver resection for malignant tumors to identify patients with sarcopenia to minimize the risk of morbidity.

formed, intraoperative data (Pringle maneuver, blood loss, blood transfusions, and operative time), and postoperative outcomes (90-day mortality, 90-day morbidity, and hospital stay) were prospectively recorded. The following patients were excluded: (1) those with benign lesions, (2) those undergoing exploratory laparotomy or laparoscopy without liver resection, (3) those undergoing extrahepatic resection, and (4) those with no preoperative computed tomographic (CT) scan.

The primary outcome of this prospective cohort study was 90-day morbidity. As secondary outcomes, 90-day mortality, hospital stay, and readmission rate were investigated. This study followed the Consolidated Standards of Reporting Trials (CONSORT) reporting guideline.

The trial protocol (Supplement 1) conforms to the ethical guidelines of the Declaration of Helsinki ${ }^{35}$ and was approved by the local institutional review board (Comitato Etico Lazio 1 Azienda Ospedaliera San Camillo Forlanini, Rome, Italy). Every case was discussed in a multidisciplinary setting, and written informed consent was obtained from each patient.

\section{Sarcopenia and Biometric Parameters}

In addition to the demographic characteristics, further biometric parameters were measured for each patient. Muscle mass was measured and calculated at the level of the third lumbar vertebra on the most recent CT scan (a maximum of 1 month from the date of the images and the date of surgery). At this level, skeletal muscles were identified and quantified by a Hounsfield unit interval of -29 to +150 ; measurements were calibrated with water and air at fixed intervals, excluding both vasculature and fatty infiltration. Cross-sectional areas (in centimeters squared) of skeletal muscles were measured by manual outlining on the CT images (Figure 1) and normalized for height to obtain the skeletal muscle index (SMI). SliceOmatic software, version 5.0 (TomoVision) was used for calculations, and 2 of us (G.B. and G.A.) independently scored each patient in a blinded fashion to obtain a mean SMI. A receiver operating characteristic curve using morbidity as a marker of sensitivity and specificity was used to identify the threshold for reduced muscle mass in the study population. Visceral adipose tissue was also assessed by manual outlining on the SliceOmatic software using a Hounsfield unit interval of -150 to -50 . As a further quantitative analysis, tricipital skinfold and subscapular skinfold were measured at hospital admission using a skinfold caliper. ${ }^{21,22}$ 
Figure 1. Computed Tomographic Scans Showing Areas of Visceral Adipose Tissue and Skeletal Muscle Mass in Patients Without Sarcopenia and Patients With Sarcopenia

A Patients without sarcopenia

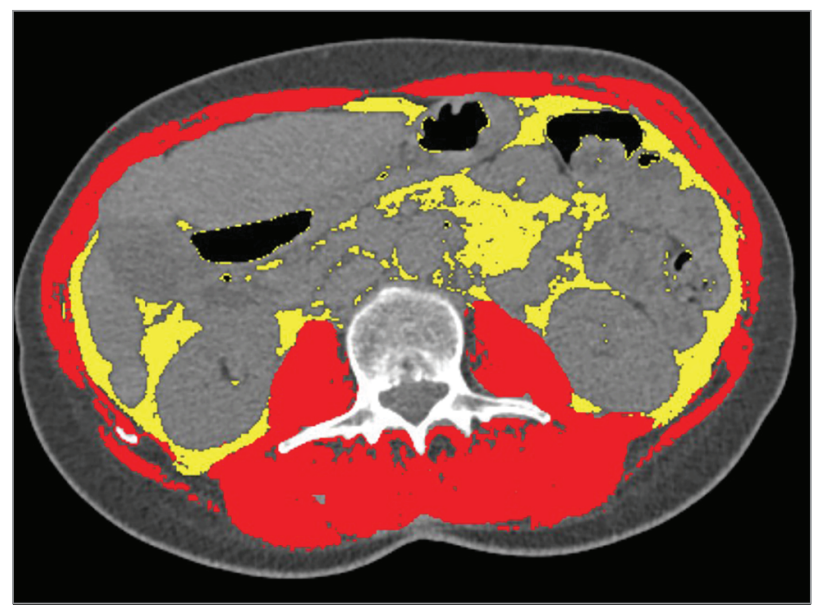

B Patients with sarcopenia

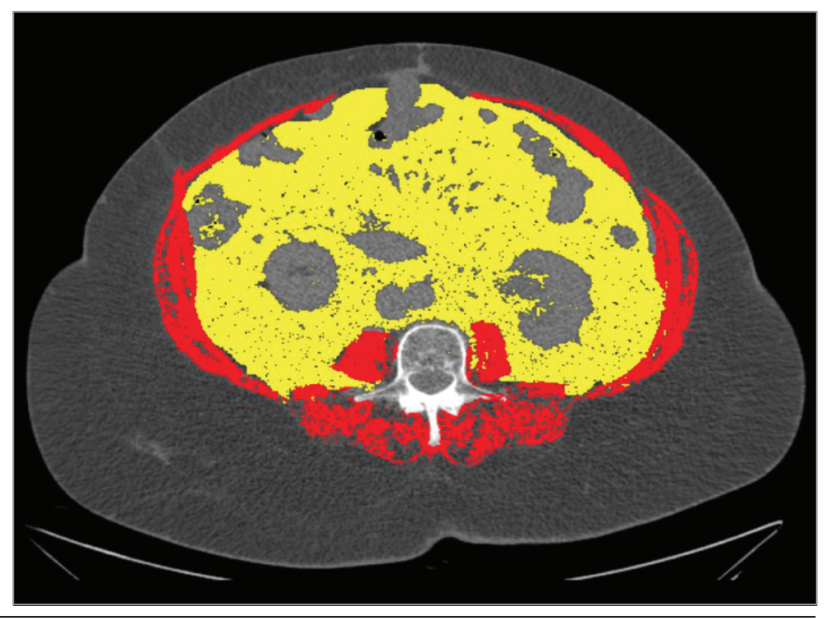

$A$ and $B$, Yellow indicates visceral adipose tissue, and red indicates skeletal muscle mass.

To evaluate muscle strength, the handgrip strength test was performed in all patients the day before surgery using a dynamometer. ${ }^{23}$ Two measurements were taken from each hand, and the average of 4 values (in kilograms) was obtained. A cutoff of less than $30 \mathrm{~kg}$ for men and less than $20 \mathrm{~kg}$ for women was used to define patients with reduced muscle strength as reported elsewhere. ${ }^{24}$ In a further analysis, the gait speed test was performed (in meters per second) using a 10-m straight path. ${ }^{25}$

Patients were then divided into 4 groups based on the presence of sarcopenia using the SMI and results of the handgrip strength test. Group A had normal muscle mass and strength, group B had reduced muscle strength, group $C$ had reduced muscle mass, and group D had reduced muscle mass and strength.

\section{Definitions}

Sarcopenia was defined by reduced muscle mass and strength as recommended recently by the European Working Group on Sarcopenia in Older People (EWGSOP). ${ }^{26}$ Portal hypertension was defined by the radiological presence of substantial splenomegaly, umbilical vein recanalization or portosystemic shunt, or preoperative platelet count less than $100 \times 10^{3} / \mu \mathrm{L}$ (to convert platelet count to $\times 10^{9}$ per liter, multiply by 1.0$) .{ }^{27}$ If the hepatic venous pressure gradient was available, a cutoff of $10 \mathrm{~mm} \mathrm{Hg}$ was considered clinically significant portal hypertension. ${ }^{28}$ Patient comorbidities were graded using the Charlson Comorbidity Index score. ${ }^{29}$ Major liver resections were considered the resection of 3 or more segments.

Morbidity was graded according to the Clavien-Dindo classification $^{30}$ and the comprehensive complication index. ${ }^{31}$ Postoperative ascites was considered a drainage output exceeding $10 \mathrm{~mL} / \mathrm{kg}$ of preoperative body weight in 24 hours. $^{32}$ Posthepatectomy liver failure and bile leakage were assessed according to definitions by the International Study Group of Liver Surgery..$^{33,34}$
Perioperative Management of Patients

All patients with liver tumors referred to us are discussed in a multidisciplinary meeting involving hepatobiliary surgeons, hepatologists, medical oncologists, radiologists, and pathologists. Before surgery, comorbidities, liver function, bleeding, and thrombotic risk are assessed and managed accordingly. For major hepatectomies, CT scan volumetry is performed to assess the future liver remnant in an effort to estimate the risk of posthepatectomy liver failure. The future liver remnant volume percentage is calculated by dividing the future liver remnant volume (in milliliters) by the total functional liver volume. A cutoff of $20 \%$ for normal livers and a cutoff of $30 \%$ to $40 \%$ for postchemotherapy and cirrhotic livers are considered safe hepatectomies. All surgical procedures are performed under standard general anesthesia with invasive arterial and central venous pressure monitoring. During resection, fluid transfusion is restricted to obtain a central venous pressure of less than $5 \mathrm{~mm} \mathrm{Hg}$. Fluids are then restored at the end of the parenchymal transection. For both open and laparoscopic procedures, we apply a standardized fast-track protocol, including removal of the central line, nasogastric tube, and urinary catheter on postoperative day (POD) 1; early mobilization (POD 1); gradual diet advancement (full diet on POD 3-4); step-down switch to oral analgesia (POD 3); and early removal of drains (POD 3-4). Complete blood testing is performed on POD 1, 3, and 5. Patients are discharged home when adequate mobilization, toleration of a solid diet, and pain control with oral medication are achieved.

\section{Statistical Analysis}

The distribution of variables was assessed using the Kolmogorov-Smirnov test and the Shapiro-Wilk test. Data are expressed as the mean (SD) for parametric continuous variables and as the median (interquartile range [IQR]) for nonparametric distribution of data. Categorical data are expressed as a number (percentage). 


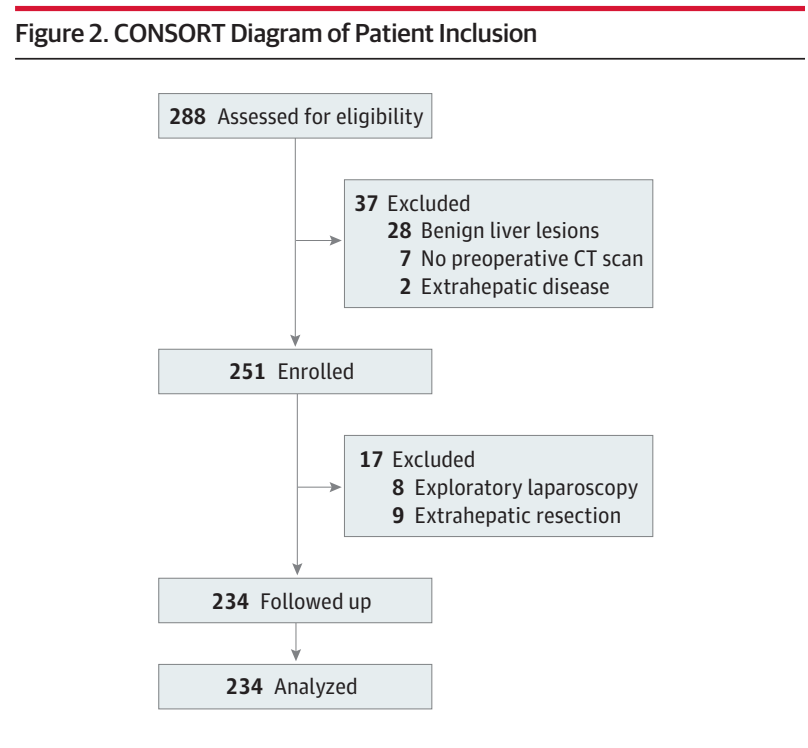

$\mathrm{CT}$ indicates computed tomographic.

The $\chi^{2}$ test or Fisher exact test with Yates correction was used to compare differences in categorical variables when appropriate. Analysis of variance was used to compare continuous parametric variables, and the Kruskal-Wallis test was used for continuous nonparametric variables. Post hoc analysis was performed using Bonferroni adjustment in the case of statistically significant differences observed among multiple groups.

To estimate the probability of morbidity, multivariable logistic regression was performed that included statistically significant risk factors in the univariable analysis. Model selection was performed according to the lowest Akaike information criterion and bayesian information criterion. Statistical analysis was performed using SPSS software, version 20.0 (IBM Corp) for MacOSX. Two-sided $P<.05$ was considered statistically significant.

\section{Results}

In total, 288 patients were assessed for eligibility during the study period (Figure 2). Excluded were 28 patients with benign liver lesions and 2 with extrahepatic tumor spread. In addition, 7 patients were excluded because no preoperative CT scan was available, 8 patients because surgery was canceled after exploratory laparoscopy, and 9 patients because extrahepatic resection was carried out. In total, 234 patients underwent 64 major (27.4\%) and 170 minor (72.6\%) hepatectomies (median age, 66.50 [IQR, 58.00-74.25] years; 158 men [67.5\%] and 76 women [32.5\%]). The median BMI (calculated as weight in kilograms divided by height in meters squared) was 27.12 (IQR, 23.28-29.55), and the most common indication for surgery was hepatocellular carcinoma (101 [43.2\%]) followed by colorectal liver metastases (96 [41.0\%]). In total, 163 patients (69.7\%) had comorbidities, 89 patients (38.0\%) had liver cirrhosis, and 88 patients (37.6\%) received a median of 8 (IQR, 6-11) cycles of neoadjuvant chemotherapy. At 90 days after hepatectomy, 5 of 234 patients (2.1\%) had died, and 72 of 234 patients (30.8\%) developed postoperative 90 -day morbidity. The rate of major morbidity was $7.7 \%$ (18 of 234).

Subgroup Analysis According to the Presence of Sarcopenia The median SMI of the entire population was 46.22 (IQR, 38.60$58.20) \mathrm{cm} / \mathrm{m}^{2}$. Based on the receiver operating characteristic curve analysis, the thresholds for defining patients with reduced muscle mass were $53.5 \mathrm{~cm} / \mathrm{m}^{2}$ for men and $40.8 \mathrm{~cm} / \mathrm{m}^{2}$ for women. The median handgrip strength of the entire population was 30.80 (IQR, 22.30-36.90) kg, with 39 of 158 men (24.7\%) performing less than $30 \mathrm{~kg}$ and 41 of 76 women (53.9\%) performing less than $20 \mathrm{~kg}$. Based on the SMI and the handgrip strength test, patients were divided into the 4 groups (Table 1). Patients with reduced muscle mass and strength (group D) had a statistically significantly lower median SMI compared with patients in the other groups (37.14 [IQR, 33.0642.47] vs 43.77 [IQR, $38.12-48.16$ ] $\mathrm{cm} / \mathrm{m}^{2}$ in group C, 57.07 [IQR, $52.51-59.46$ ] cm $/ \mathrm{m}^{2}$ in group $B$, and 60.00 [IQR, 56.92$60.00] \mathrm{cm} / \mathrm{m}^{2}$ in group A; $P<.001$ ), decreased visceral adipose tissue (93.15 [IQR, 44.00-154.20] vs 170.60 [IQR, 89.30$229.55] \mathrm{cm}^{2}$ in group C, 198.50 [IQR, 19.60-270.60] $\mathrm{cm}^{2}$ in group B, and 269.50 [IQR, 164.52-323.90] $\mathrm{cm}^{2}$ in group A; $P<.001)$, and weaker handgrip strength (19.20 [IQR, 18.2022.40 ] vs 35.40 [IQR, 31.00-38.70] kg in group C, 27.30 [IQR, 18.95-29.10] kg in group B, and 34.00 [IQR, 30.00-39.77] kg in group $\mathrm{A} ; P<.001$ ) (Figure 3 ). No differences in BMI, tricipital skinfold, subscapular skinfold, and gait speed were observed among the groups.

Regarding postoperative results, patients with reduced muscle mass and strength (group D) had a statistically significantly higher rate of 90-day morbidity compared with patients in the other groups (51.5\% [35 of 68] in group D vs 38.7\% [29 of 75] in group C, 23.1\% [3 of 13] in group B, and 6.4\% [5 of 78 ] in group $\mathrm{A} ; P<.001)$ and a higher median comprehensive complication index (60.45 [IQR, 20.90-82.00] vs 20.90 [IQR, 20.90-20.90] in group C, 20.90 [IQR, 20.90-33.75] in group $B$, and 2.90 [IQR, 2.90-2.90] in group A; $P=.02$ ) (Table 2). Although not statistically significant, the rate of major morbidity (Clavien-Dindo classification III-IV) was also higher in group $\mathrm{D}$ than in the other groups (17.6\% [12 of 68] in group D vs $6.7 \%$ [5 of 75] in group C, $0 \%$ [O of 13] in group B, and $1.3 \%$ [1 of 78] in group $\mathrm{A} ; P=.30)$. In addition, the median hospital stay was longer in group D (10 [IQR, 7.00-15.00] days in group D vs 8 [IQR, 5.00-8.00] days in group C, 9 [IQR, 6.00-9.00] days in group $B$, and 6 [IQR, 5.00-7.50] days in group A; $P<.001$ ), and more patients in group $\mathrm{D}$ were readmitted to the hospital after discharge (8.8\% [6 of 68] in group D vs 5.3\% [4 of 75] in group C, $7.7 \%$ [ 1 of 13 ] in group $\mathrm{B}$, and $0 \%$ [0 of 78] in group A; $P=.02)$.

\section{Risk Factors Associated With Morbidity}

According to the results of the multivariable logistic regression, the following factors were statistically significantly associated with an increased risk of postoperative 90-day morbidity in the univariable analysis: American Society of 


\begin{tabular}{|c|c|c|c|c|c|c|}
\hline \multirow[b]{2}{*}{ Variable } & \multicolumn{5}{|l|}{ Median (IQR) } & \multirow[b]{2}{*}{$P$ value } \\
\hline & Total $(\mathrm{N}=234)$ & $\begin{array}{l}\text { Group A: normal } \\
\text { muscle mass and } \\
\text { strength }(n=78)\end{array}$ & $\begin{array}{l}\text { Group B: reduced } \\
\text { muscle strength } \\
(\mathrm{n}=13)\end{array}$ & $\begin{array}{l}\text { Group C: reduced } \\
\text { muscle mass } \\
(\mathrm{n}=75)\end{array}$ & $\begin{array}{l}\text { Group D: reduced } \\
\text { muscle mass and } \\
\text { strength }(n=68)\end{array}$ & \\
\hline Age, y & $\begin{array}{l}66.50 \\
(58.00-74.25)\end{array}$ & $\begin{array}{l}68.00 \\
(59.75-74.00)\end{array}$ & $\begin{array}{l}66.00 \\
(60.00-73.50)\end{array}$ & $\begin{array}{l}66.00 \\
(60.00-74.00)\end{array}$ & $68.00(54.50-75.00)$ & .90 \\
\hline \multicolumn{7}{|l|}{ Sex, No. } \\
\hline Female & 76 & 22 & 3 & 14 & 37 & \multirow{2}{*}{$<.001$} \\
\hline Male & 158 & 56 & 10 & 61 & 31 & \\
\hline ASA score & $2.00(2.00-2.00)$ & $2.00(2.00-2.00)$ & $2.00(2.00-2.00)$ & $2.00(2.00-2.00)$ & $2.00(2.00-2.00)$ & .84 \\
\hline Performance status ${ }^{a}$ & $100(100-100)$ & $100(100-100)$ & $100(100-100)$ & $100(100-100)$ & $100(100-100)$ & .09 \\
\hline Comorbidities, №. (\%) & $163(69.7)$ & $56(71.8)$ & $9(69.2)$ & $57(76.0)$ & $41(60.3)$ & .22 \\
\hline Charlson Comorbidity Index score & $4.00(3.00-6.00)$ & $5.00(3.00-6.00)$ & $3.00(3.00-5.50)$ & $5.00(3.00-6.00)$ & $3.00(2.00-5.00)$ & .42 \\
\hline BMI & $\begin{array}{l}27.12 \\
(23.28-29.55)\end{array}$ & $\begin{array}{l}27.10 \\
(22.26-30.32)\end{array}$ & $\begin{array}{l}28.10 \\
(22.70-31.75)\end{array}$ & $\begin{array}{l}26.34 \\
(25.62-29.70)\end{array}$ & $25.06(23.40-31.18)$ & .11 \\
\hline Skeletal muscle index, $\mathrm{cm} / \mathrm{m}^{2}$ & $\begin{array}{l}46.22 \\
(38.60-58.20)\end{array}$ & $\begin{array}{l}60.00 \\
(56.92-60.00)\end{array}$ & $\begin{array}{l}57.07 \\
(52.51-59.46)\end{array}$ & $\begin{array}{l}43.77 \\
(38.12-48.16)\end{array}$ & $37.14(33.06-42.47)$ & $<.001$ \\
\hline Visceral adipose tissue, $\mathrm{cm}^{2}$ & $\begin{array}{l}150.90 \\
(71.37-224.17)\end{array}$ & $\begin{array}{l}269.50 \\
(164.52-323.90)\end{array}$ & $\begin{array}{l}198.50 \\
(19.60-270.60)\end{array}$ & $\begin{array}{l}170.60 \\
(89.30-229.55)\end{array}$ & $\begin{array}{l}93.15 \\
(44.00-154.20)\end{array}$ & $<.001$ \\
\hline Tricipital skinfold, mm & $\begin{array}{l}16.00 \\
(11.00-22.00)\end{array}$ & $\begin{array}{l}16.00 \\
(10.00-23.00)\end{array}$ & $\begin{array}{l}15.00 \\
(13.00-21.50)\end{array}$ & $\begin{array}{l}15.00 \\
(12.00-20.00)\end{array}$ & $16.00(12.00-24.50)$ & .78 \\
\hline Subscapular skinfold, mm & $\begin{array}{l}17.00 \\
(12.00-21.00)\end{array}$ & $\begin{array}{l}17.00 \\
(17.00-22.00)\end{array}$ & $\begin{array}{l}17.00 \\
(15.00-21.50)\end{array}$ & $\begin{array}{l}18.00 \\
(12.00-21.00)\end{array}$ & $15.50(10.00-21.00)$ & .44 \\
\hline Handgrip strength, kg & $\begin{array}{l}30.80 \\
(22.30-36.90)\end{array}$ & $\begin{array}{l}34.00 \\
(30.00-39.77)\end{array}$ & $\begin{array}{l}27.30 \\
(18.95-29.10)\end{array}$ & $\begin{array}{l}35.40 \\
(31.00-38.70)\end{array}$ & $19.20(18.20-22.40)$ & $<.001$ \\
\hline Gait speed, $\mathrm{m} / \mathrm{s}$ & $1.00(0.97-1.20)$ & $1.09(1.00-1.20)$ & $1.09(1.00-1.18)$ & $1.00(0.90-1.12)$ & $1.00(0.90-1.10)$ & .07 \\
\hline \multicolumn{7}{|l|}{ Cause, No. (\%) } \\
\hline Hepatocellular carcinoma & $101(43.2)$ & $38(48.7)$ & $7(53.8)$ & $36(48.0)$ & $20(29.4)$ & \multirow{5}{*}{.008} \\
\hline Colorectal liver metastases & $96(41.0)$ & $34(43.6)$ & $1(7.7)$ & $29(38.7)$ & $32(47.1)$ & \\
\hline Cholangiocarcinoma & $30(12.8)$ & $3(3.8)$ & $4(30.8)$ & $9(12.0)$ & $14(20.6)$ & \\
\hline Gallbladder cancer & $4(1.7)$ & $2(2.6)$ & $1(7.7)$ & $1(1.3)$ & 0 & \\
\hline Noncolorectal liver metastases & $3(1.3)$ & $1(1.3)$ & 0 & 0 & $2(2.9)$ & \\
\hline Portal hypertension, №. (\%) & $23(9.8)$ & $5(6.4)$ & $2(15.4)$ & $9(12.0)$ & $7(10.3)$ & .23 \\
\hline Liver cirrhosis, No. (\%) & $89(38.0)$ & $25(32.1)$ & $7(53.8)$ & $33(44.0)$ & $24(35.3)$ & .27 \\
\hline \multicolumn{7}{|l|}{ Child-Pugh score, No. (\%) } \\
\hline A & $79(33.8)$ & $22(28.2)$ & $7(53.8)$ & $28(37.3)$ & $22(32.4)$ & \multirow{2}{*}{.42} \\
\hline B & $4(1.7)$ & $2(2.6)$ & 0 & $2(2.7)$ & 0 & \\
\hline MELD score & $8.00(7.00-9.00)$ & $8.00(7.00-9.00)$ & $8.00(6.50-9.00)$ & $8.00(7.00-8.75)$ & $8.00(5.50-9.00)$ & .88 \\
\hline Neoadjuvant chemotherapy, №. (\%) & $88(37.6)$ & $31(39.7)$ & $5(38.5)$ & $23(30.7)$ & $29(42.6)$ & .48 \\
\hline Preoperative hemoglobin, g/dL & $\begin{array}{l}13.00 \\
(12.00-14.20)\end{array}$ & $\begin{array}{l}12.90 \\
(12.00-14.00)\end{array}$ & $\begin{array}{l}15.00 \\
(12.70-15.50)\end{array}$ & $\begin{array}{l}13.50 \\
(12.00-14.70)\end{array}$ & $12.25(10.52-13.50)$ & .001 \\
\hline Preoperative creatinine, $\mathrm{mg} / \mathrm{dL}$ & $0.90(0.80-1.00)$ & $0.90(0.80-1.00)$ & $1.10(0.90-1.25)$ & $0.80(0.80-1.00)$ & $0.89(0.70-1.00)$ & .009 \\
\hline Preoperative albumin, $\mathrm{g} / \mathrm{dL}$ & $4.10(3.70-4.10)$ & $3.90(3.40-4.23)$ & $4.30(4.10-4.50)$ & $4.10(3.70-4.40)$ & $4.37(3.92-4.65)$ & .04 \\
\hline Preoperative AST level, U/L & $\begin{array}{l}30.00 \\
(23.00-50.00)\end{array}$ & $\begin{array}{l}33.50 \\
(23.75-51.00)\end{array}$ & $\begin{array}{l}34.00 \\
(30.00-69.00)\end{array}$ & $\begin{array}{l}29.50 \\
(23.00-56.00)\end{array}$ & $26.00(21.50-35.00)$ & .62 \\
\hline Preoperative ALT level, U/L & $\begin{array}{l}28.00 \\
(19.00-45.25)\end{array}$ & $\begin{array}{l}31.50 \\
(19.00-42.00)\end{array}$ & $\begin{array}{l}36.00 \\
(26.50-72.00)\end{array}$ & $\begin{array}{l}28.00 \\
(18.75-55.25)\end{array}$ & $39.65(25.59-53.71)$ & .12 \\
\hline Preoperative GGT level, U/L & $\begin{array}{l}53.00 \\
(26.50-154.00)\end{array}$ & $\begin{array}{l}50.00 \\
(25.00-123.00)\end{array}$ & $\begin{array}{l}131.00 \\
(47.00-248.50)\end{array}$ & $\begin{array}{l}50.00 \\
(27.75-149.00)\end{array}$ & $24.00(17.00-38.00)$ & .07 \\
\hline Preoperative bilirubin level, $\mathrm{mg} / \mathrm{dL}$ & $0.90(0.60-1.10)$ & $0.95(0.60-1.17)$ & $1.00(0.90-1.10)$ & $0.80(0.60-1.20)$ & $0.60(0.50-0.90)$ & .57 \\
\hline Preoperative INR & $1.10(1.02-1.18)$ & $1.10(1.00-1.20)$ & $1.10(1.02-1.17)$ & $1.08(1.03-1.15)$ & $1.10(1.01-1.17)$ & .65 \\
\hline Preoperative platelet count, $\times 10^{3} / \mu \mathrm{L}$ & $188(134-248)$ & $184(123-244)$ & $153(122-211)$ & $177(132-243)$ & $205(160-276)$ & .02 \\
\hline Lesion size, $\mathrm{mm}$ & $\begin{array}{l}35.00 \\
(25.00-60.00)\end{array}$ & $\begin{array}{l}30.00 \\
(20.00-60.00)\end{array}$ & $\begin{array}{l}41.00 \\
(24.25-60.00)\end{array}$ & $\begin{array}{l}31.50 \\
(26.75-47.00)\end{array}$ & $46.00(30.00-67.50)$ & .04 \\
\hline No. of lesions & $1.00(1.00-2.00)$ & $1.00(1.00-3.00)$ & $1.00(1.00-1.00)$ & $1.00(1.00-2.00)$ & $1.00(100-2.00)$ & .15 \\
\hline \multicolumn{3}{|c|}{$\begin{array}{l}\text { Abbreviations: ALT, alanine aminotransferase; ASA, American Society of } \\
\text { Anesthesiologists; AST, aspartate aminotransferase; BMI, body mass index } \\
\text { (calculated as weight in kilograms divided by height in meters squared); } \\
\text { GGT, y-glutamyltransferase; INR, international normalized ratio; } \\
\text { IQR, interquartile range; MELD, model for end-stage liver disease. }\end{array}$} & \multicolumn{4}{|c|}{$\begin{array}{l}\text { ALT level to microkatals per liter, multiply by } 0.0167 \text {; AST level to microkatals } \\
\text { per liter, multiply by } 0.0167 \text {; bilirubin level to micromoles per liter, multiply by } \\
\text { 17.104; creatinine level to micromoles per liter, multiply by } 88.4 \text {; GGT level to } \\
\text { microkatals per liter, multiply by } 0.0167 \text {; hemoglobin level to grams per liter, } \\
\text { multiply by } 10.0 \text {; and platelet count to } \times 10^{9} / \mathrm{L} \text {, multiply by } 1.0 \text {. }\end{array}$} \\
\hline \multicolumn{3}{|c|}{ SI conversion factors: To convert albumin level to grams per liter, multiply by 10; } & a Eastern Cooperativ & Oncology Group sco & e (range, $1[$ low] to $100[\mathrm{l}$ & [high]). \\
\hline
\end{tabular}


Figure 3. Skeletal Muscle Index, Handgrip Strength Test, Visceral Adipose Tissue, and BMI According to Muscle Mass and Muscle Strength

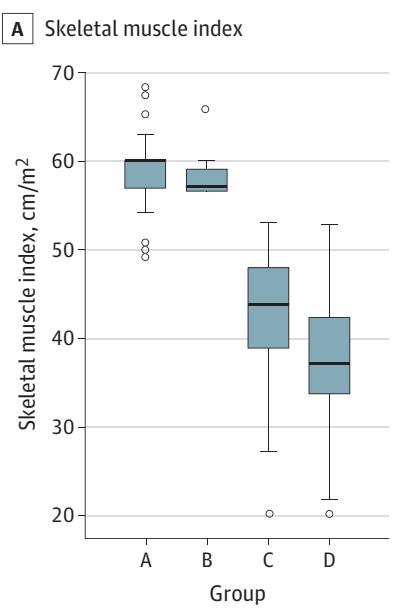

B Handgrip strength test
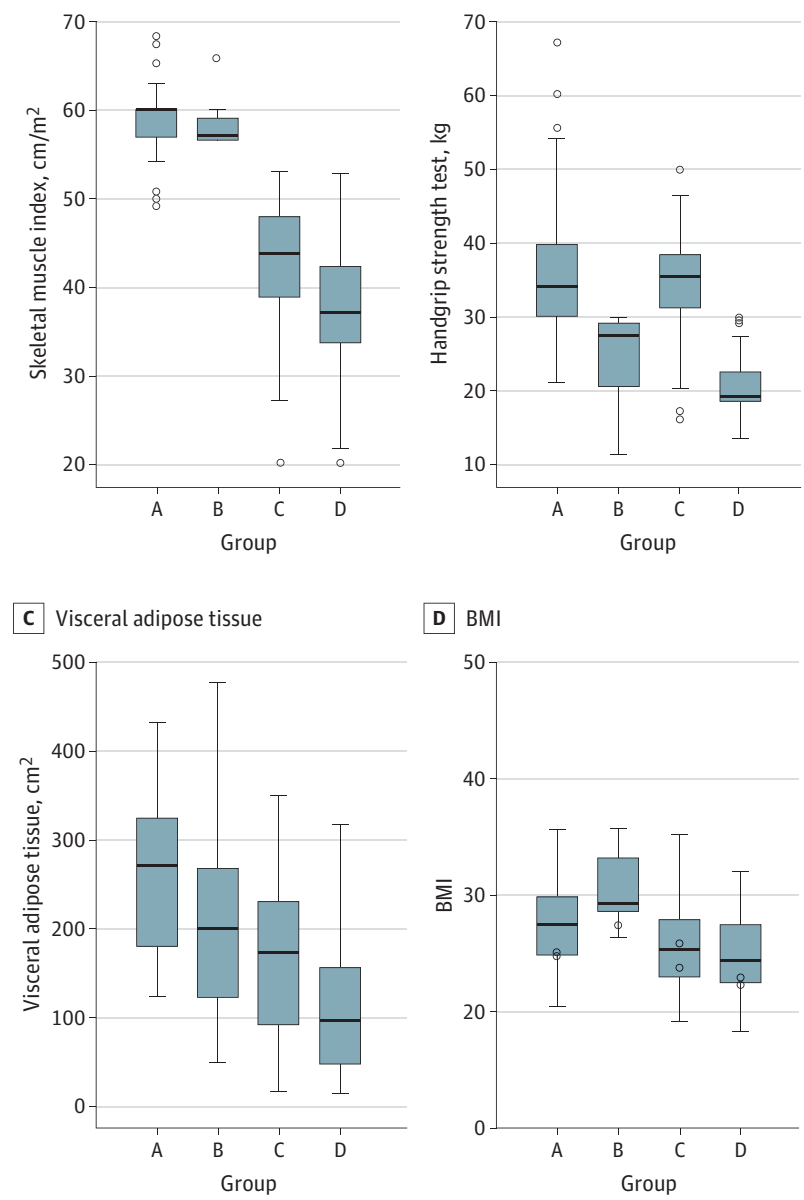

A-D, The median (interquartile range) is shown. Group A is normal muscle mass and strength, group $B$ is reduced muscle strength, group $C$ is reduced muscle mass, and group $D$ is reduced muscle mass and strength. BMI indicates body mass index (calculated as weight in kilograms divided by height in meters squared).

Anesthesiologists score, SMI, handgrip strength, sarcopenia, liver cirrhosis, preoperative portal hypertension, open surgery approach, major hepatectomy, biliary reconstruction, blood loss, operative time, and creatinine level (eTable in Supplement 2). In the multivariable analysis, sarcopenia (hazard ratio, 3.92; 95\% CI, 1.96-7.80; $P<.001$ ), liver cirrhosis (hazard ratio, 3.63; 95\% CI, 1.44-9.15; $P=.006)$, portal hypertension (hazard ratio, 5.88; 95\% CI, 1.83-18.89; $P=.003$ ), and biliary reconstruction (hazard ratio, 6.00; 95\% CI, 1.6022.43; $P=.008$ ) were independent risk factors associated with 90-day morbidity. Among 89 patients with liver cirrhosis (38.0\%), those with reduced muscle mass and strength (group D) had a statistically significantly higher rate of postoperative 90 -day morbidity (87.5\% [21 of 24 ] in group D vs $72.7 \%$ [24 of 33] in group C, $28.6 \%$ [ 2 of 7] in group B, and $4.0 \%$ [1 of $25]$ in group $\mathrm{A} ; P<.001)$.

\section{Discussion}

To our knowledge, this study is the first prospective cohort study reporting that patients with sarcopenia are at higher risk of developing postoperative 90-day morbidity after liver resection for malignant tumors. Patients with cancer are frequently older individuals with comorbidities, cachexia, and limited functional reserve. Furthermore, in the case of malignant tumors of the liver, patients frequently have signs of concomitant liver cirrhosis and may have undergone neoadjuvant chemotherapy. Almost 70\% of patients in our study had comorbidities, $38.0 \%$ had liver cirrhosis, and 37.6\% were referred after neoadjuvant chemotherapy. Therefore, alterations in general and liver-specific function increased surgical risk.

Liver resections are complex procedures burdened by the risk of postoperative morbidity. In our consecutive series of patients, the overall 90-day mortality and morbidity rates were $2.1 \%$ and $30.8 \%$, respectively, in line with the available literature ${ }^{4-6}$ but still an issue to consider in hepatobiliary surgery. In this study, we observed that patients without sarcopenia had statistically significantly better outcomes than patients with sarcopenia. Because surgical morbidity is associated with longer hospitalizations and higher medical costs, it is important to identify patients undergoing hepatic resection who are at high risk for postoperative morbidity. ${ }^{15}$ Because many risk factors are not modifiable (eg, age, comorbidities, and type of surgery), our focus should be on factors that could be modified or ameliorated before surgery. The presence of sarcopenia is of great interest because it is associated with worse outcomes and may be addressed before surgery. In fact, numerous studies ${ }^{36-42}$ have focused on treating sarcopenia and the associated metabolic imbalances before surgery.

Published data also suggest that exercise and dietary supplementation might substantially improve muscle mass in patients with cancer (specifically, 30-40 minutes of exercise 3-4 times a week or 2000 extra steps daily, with the addition of late evening snacks containing branched-chain amino acids, for approximately 3 months). ${ }^{20,36,37}$ Such intervention seems promising because it could substantially alter both short-term and long-term outcomes after cancer surgery. However, delaying surgical intervention in patients with cancer may worsen prognosis and result in tumor progression. Among patients in whom surgery is scheduled after neoadjuvant chemotherapy (eg, for colorectal liver metastases or cholangiocarcinoma) or after locoregional treatment (eg, for hepatocellular carcinoma), exercise and dietary supplementation might be implemented before surgery. However, neoadjuvant chemotherapy is also associated with muscle mass loss and worsening body composition in patients with colorectal liver metastases. ${ }^{38}$

Recently, the EWGSOP recommended that the definition of sarcopenia should be multidimensional, reflecting both muscle mass and muscle strength. ${ }^{26}$ Although quantifying sarcopenia on a single CT scan slice is the most widely adopted method, this approach does not consider appendicular muscle mass or muscle strength. Mass is only 1 of the elements comprising sarcopenia: muscles might have reduced mass but may 


\begin{tabular}{|c|c|c|c|c|c|c|}
\hline \multirow[b]{2}{*}{ Variable } & \multicolumn{5}{|l|}{ №. (\%) } & \multirow[b]{2}{*}{$P$ value } \\
\hline & Total $(\mathrm{N}=234)$ & $\begin{array}{l}\text { Group A: normal } \\
\text { muscle mass and } \\
\text { strength }(n=78)\end{array}$ & $\begin{array}{l}\text { Group B: reduced } \\
\text { muscle strength } \\
(\mathrm{n}=13)\end{array}$ & $\begin{array}{l}\text { Group C: reduced } \\
\text { muscle mass }(n=75)\end{array}$ & $\begin{array}{l}\text { Group D: reduced } \\
\text { muscle mass and } \\
\text { strength }(n=68)\end{array}$ & \\
\hline \multicolumn{7}{|l|}{ Surgical approach } \\
\hline Open & $182(77.8)$ & $57(73.1)$ & $10(76.9)$ & $55(73.3)$ & $60(88.2)$ & \multirow{2}{*}{.10} \\
\hline Laparoscopic & $52(22.2)$ & $21(26.9)$ & $3(23.1)$ & $20(26.7)$ & $8(11.8)$ & \\
\hline \multicolumn{7}{|l|}{ Type of hepatectomy } \\
\hline Major & $64(27.4)$ & $19(24.4)$ & $4(30.8)$ & $19(25.3)$ & $22(32.4)$ & \multirow{2}{*}{.69} \\
\hline Minor & $170(72.6)$ & $59(75.6)$ & $9(69.2)$ & $56(74.7)$ & $46(67.6)$ & \\
\hline Biliary reconstruction & $14(6.0)$ & $4(5.1)$ & $1(7.7)$ & $4(5.3)$ & $5(7.4)$ & .92 \\
\hline Pringle maneuver & $44(18.8)$ & $20(25.6)$ & $2(15.4)$ & $11(14.7)$ & $11(16.2)$ & .30 \\
\hline Blood loss, median (IQR), $\mathrm{cm}^{3}$ & $\begin{array}{l}150.00 \\
(100.00-250.00)\end{array}$ & $\begin{array}{l}12.00 \\
(5.00-20.00)\end{array}$ & $\begin{array}{l}200.00 \\
(150.00-400.00)\end{array}$ & $\begin{array}{l}100.00 \\
(87.50-200.00)\end{array}$ & $\begin{array}{l}180.00 \\
(100.00-300.00)\end{array}$ & .09 \\
\hline Blood transfusions & $5(2.1)$ & $1(1.3)$ & 0 & 0 & $4(5.9)$ & .08 \\
\hline Operative time, min & $\begin{array}{l}180.00 \\
(150.00-240.00)\end{array}$ & $\begin{array}{l}18.00 \\
(14.00-236.00)\end{array}$ & $\begin{array}{l}200.00 \\
(170.00-255.00)\end{array}$ & $\begin{array}{l}180.00 \\
(137.50-217.50)\end{array}$ & $\begin{array}{l}220.00 \\
(178.00-280.00)\end{array}$ & .92 \\
\hline 90-d Mortality & $5(2.1)$ & $1(1.3)$ & $1(7.7)$ & 0 & $3(4.4)$ & .13 \\
\hline 90-d Morbidity & $72(30.8)$ & $5(6.4)$ & $3(23.1)$ & $29(38.7)$ & $35(51.5)$ & $<.001^{\mathrm{a}}$ \\
\hline \multicolumn{7}{|l|}{ Clavien-Dindo classification } \\
\hline $\mid-11$ & $54(23.1)$ & $4(5.1)$ & $3(23.1)$ & $24(32.0)$ & $23(33.8)$ & \multirow{2}{*}{.30} \\
\hline III-V & $18(7.7)$ & $1(1.3)$ & 0 & $5(6.7)$ & $12(17.6)$ & \\
\hline $\begin{array}{l}\text { Comprehensive complication } \\
\text { index, median (IQR) }\end{array}$ & $\begin{array}{l}20.90 \\
(20.90-33.70)\end{array}$ & $\begin{array}{l}2.90 \\
(2.90-2.90)\end{array}$ & $\begin{array}{l}20.90 \\
(20.90-33.75)\end{array}$ & $\begin{array}{l}20.90 \\
(20.90-20.90)\end{array}$ & $\begin{array}{l}60.45 \\
(20.90-82.00)\end{array}$ & .02 \\
\hline Hospital stay, median (IQR), d & $\begin{array}{l}7.00 \\
(5.00-10.00)\end{array}$ & $\begin{array}{l}6.00 \\
(5.00-7.50)\end{array}$ & $\begin{array}{l}9.00 \\
(6.00-9.00)\end{array}$ & $\begin{array}{l}8.00 \\
(5.00-8.00)\end{array}$ & $\begin{array}{l}10.00 \\
(7.00-15.00)\end{array}$ & $<.001$ \\
\hline Readmission rate & $11(4.7)$ & 0 & $1(7.7)$ & $4(5.3)$ & $6(8.8)$ & .02 \\
\hline
\end{tabular}

maintain substantial strength (and vice versa). Moreover, preoperative muscle weakness has been previously described as an important risk factor for adverse outcomes after surgery. ${ }^{39,40}$

Following the EWGSOP criteria, ${ }^{26}$ we assessed muscle mass, handgrip strength, and gait speed for each of our patients prospectively to better characterize sarcopenia in this study. There was limited variability in gait speed among our cohort, and patients with sarcopenia often had normal gait speed. Therefore, the gait speed test has low positive predictive value. In contrast, performance on the handgrip strength test was associated with all-cause mortality in a large study ${ }^{41}$ and showed high accuracy in identifying patients with reduced muscle mass and estimating morbidity after surgery in our cohort. Subdividing patients according to the EWGSOP definition of sarcopenia, ${ }^{26}$ those with sarcopenia defined by both muscle mass and muscle strength had worse outcomes than those with sarcopenia defined by 1 measurement. Patients with sarcopenia in our study had a 3.92 times increased risk of developing postoperative morbidity compared with patients without sarcopenia. Therefore, obtaining both muscle mass and muscle strength measurements enhances our ability to identify and classify patients with sarcopenia before surgery.

Muscle strength was easily and inexpensively measured herein using the handgrip strength test with a simple dynamometer. In this setting, tests investigating muscle function, including the handgrip strength test, the gait speed test, and the chairstand test, are assessing limited muscle groups, mainly the appendicular systems, and their use may result in measurement variability and error. We do not recommend their use alone in di- agnosing sarcopenia. In contrast, the SMI is a standardized method. It allows evaluation of the composition of core muscles and abdominal fat, resulting in a value that is normalized for height based on a validated method ${ }^{43}$ with little probability of misinterpretation. Using the SMI, muscle mass and muscle strength are complementary to obtain a precise and thorough diagnosis of sarcopenia. However, because advanced software (SliceOmatic, version 5.0; TomoVision) used to identify patients with reduced muscle mass is not available in every institution, the handgrip strength test could still be an effective tool to obtain a diagnosis of probable sarcopenia. Only 3 studies to date ( 2 on gastric cancer ${ }^{44,45}$ and 1 on colorectal cancer ${ }^{46}$ ) have tested the EWGSOP definition of sarcopenia. ${ }^{26}$ All 3 studies suggested an association of sarcopenia with postoperative outcomes, which was supported in our prospective study of liver resection for malignant tumors.

The incidence of sarcopenia varies among studies depending on the populations investigated and cutoff values used. Some researchers have recommended use of the original EWGSOP definition of sarcopenia ${ }^{26}$ (SMI $<38.5 \mathrm{~cm} / \mathrm{m}^{2}$ for women and $<52.4$ $\mathrm{cm} / \mathrm{m}^{2}$ for men), as proposed by Prado et al. ${ }^{42}$ However, use of these cutoff levels may be limited because differences in races/ ethnicities and populations may alter the classification of patients. Studies using predefined cutoff levels had an incidence of sarcopenia of approximately 53\%, whereas studies using population-tailored cutoffs had an incidence of $35 \% .{ }^{11}$

In our study, we identified a population-specific SMI threshold of $53.5 \mathrm{~cm} / \mathrm{m}^{2}$ for men and $40.8 \mathrm{~cm} / \mathrm{m}^{2}$ for women to define patients with reduced muscle mass. These cutoffs are similar to 
those reported in one of the first and most important Western studies on sarcopenia and cancer cachexia ${ }^{12}$ and are much higher than those reported in a recent article by researchers in Japan. ${ }^{20}$ Therefore, our results align with those reported in previous studies, ${ }^{11,20}$ emphasizing the need for population-specific data to correctly study and predict oncological outcomes and avoid patient misclassification. Notably, the amount of visceral adipose tissue decreased in parallel to the SMI in our cohort, whereas BMI remained comparable among groups. In addition to conventional patients with cachexia, normal-weight individuals or even overweight individuals with cancer were hospitalized at San Camillo Forlanini Hospital during the study period. This finding highlights the importance of biometric calculations based on preoperative diagnostic images, providing additional important insights and possibly improving patient selection.

\section{Strengths and Limitations}

This investigation is the first prospective cohort study to date to evaluate the association of sarcopenia with outcomes after liver resection for malignant tumors. Both muscle mass and strength measures were gathered prospectively in an effort to reduce selection bias, which has not been done previously, to our knowledge. The sample size was assessed a priori and was reached in 18 months because the study was conducted in a high-volume center performing more than 250 liver resections per year. This strength avoids the limitations of previ- ous retrospective studies ${ }^{11}$ that evaluated sarcopenia over 10 to 15 years, which may have resulted in selection bias and failure to control for recent advancements in chemotherapies, surgical techniques, and patient management.

This study has limitations. We included both patients with liver cirrhosis and patients without liver cirrhosis. Although these groups were well balanced, different grades of liver cirrhosis and varied degrees of portal hypertension might have introduced selection bias and impacted our outcomes.

\section{Conclusions}

Sarcopenia is associated with postoperative results after liver resection for both primary and secondary hepatic malignant tumors. When evaluating patients with sarcopenia, our data from a single center suggest that both muscle mass measurements on preoperative CT scans and muscle strength assessments with the handgrip strength test should be obtained at the first clinical encounter to better classify patients and to minimize the risk of morbidity. In addition, the inclusion of sarcopenia evaluation in routine clinical practice may be beneficial to address patient informed consent and to correct adverse preoperative conditions if possible. Future research is needed on the observed association between sarcopenia modification and postoperative outcomes.

\section{ARTICLE INFORMATION}

Accepted for Publication: May 23, 2020.

Published Online: September 23, 2020. doi:10.1001/jamasurg.2020.3336

Author Affiliations: Department of General Surgery and Liver Transplantation, San Camillo Forlanini Hospital, Rome, Italy (Berardi, Colasanti, Meniconi, Guglielmo, Laurenzi, Ferretti, Levi Sandri, Ettorre); Department of Digestive and Liver Diseases, University Sapienza, Rome, Italy (Antonelli, Marignani); Statistics Section, Department of Public Health and Infectious Diseases, University Sapienza, Rome, Italy (Spagnoli); Department of Radiology, San Camillo Forlanini Hospital, Rome, Italy (Moschetta, Schininà, Antonini); Department of General Surgery, San Camillo Forlanini Hospital, Rome, Italy (Moschetta); Department of Anesthesiology and Intensive Care, San Camillo Forlanini Hospital, Rome, Italy (Antonini).

Author Contributions: Drs Berardi and Antonelli contributed equally to this work. Drs Berardi and Antonelli had full access to all of the data in the study and take responsibility for the integrity of the data and the accuracy of the data analysis. Concept and design: Berardi, Antonelli, Colasanti, Ferretti, Levi Sandri, Spagnoli, Schininà, Marignani, Ettorre.

Acquisition, analysis, or interpretation of data: Berardi, Antonelli, Meniconi, Guglielmo, Laurenzi, Ferretti, Spagnoli, Moschetta, Antonini. Drafting of the manuscript: Berardi, Antonelli, Colasanti, Ferretti, Spagnoli, Moschetta. Critical revision of the manuscript for important intellectual content: Berardi, Antonelli, Meniconi, Guglielmo, Laurenzi, Ferretti, Levi Sandri, Spagnoli, Schininà, Antonini, Marignani, Ettorre.
Statistical analysis: Colasanti, Spagnoli, Moschetta. Administrative, technical, or material support: Antonelli, Guglielmo, Laurenzi, Ferretti, Levi Sandri. Supervision: Berardi, Antonelli, Colasanti, Meniconi, Laurenzi, Ferretti, Schininà, Antonini, Marignani, Ettorre.

Conflict of Interest Disclosures: None reported.

Additional Contributions: Quinn W. McCurren, MS (San Camillo Forlanini Hospital, Rome, Italy). assisted with English-language editing of the manuscript. He was not compensated for his contributions. The following individuals contributed to this study: Paola Begini (Department of Gastroenterology and Hepatology, University Sapienza, Rome, Italy) and Mirco Burocchi, Alessandra Campanelli, Federica Falaschi, Imma Iannone, Tatiana Leggeri, Davide Rossi, Luca Saccco, Andrea Scotti, and Giuditta Serrao (Department of General Surgery and Liver Transplantation, San Camillo Forlanini Hospital, Rome, Italy). They were not compensated for their contributions.

\section{REFERENCES}

1. Cohen DJ, Leichman L. Controversies in the treatment of local and locally advanced gastric and esophageal cancers. J Clin Oncol. 2015;33(16):1754 1759. doi:10.1200/JCO.2014.59.7765

2. Heestand GM, Murphy JD, Lowy AM. Approach to patients with pancreatic cancer without detectable metastases. J Clin Oncol. 2015;33(16): 1770-1778. doi:10.1200/JCO.2014.59.7930

3. Knox JJ, Cleary SP, Dawson LA. Localized and systemic approaches to treating hepatocellular carcinoma. J Clin Oncol. 2015;33(16):1835-1844. doi:10.1200/JCO.2014.60.1153
4. Belghiti J, Hiramatsu $K$, Benoist $S$, Massault $P$, Sauvanet A, Farges O. Seven hundred forty-seven hepatectomies in the 1990s: an update to evaluate the actual risk of liver resection. J Am Coll Surg. 2000;191(1):38-46. doi:10.1016/S1072-7515(00) 00261-1

5. Kamiyama T, Nakanishi K, Yokoo H, et al. Perioperative management of hepatic resection toward zero mortality and morbidity: analysis of 793 consecutive cases in a single institution. J Am Coll Surg. 2010;211(4):443-449. doi:10.1016/j. jamcollsurg.2010.06.005

6. Virani S, Michaelson JS, Hutter MM, et al. Morbidity and mortality after liver resection: results of the Patient Safety in Surgery Study. J Am Coll Surg 2007;204(6):1284-1292. doi:10.1016/j.jamcollsurg. 2007.02.067

7. Bhangui P, Laurent A, Amathieu R, Azoulay D. Assessment of risk for non-hepatic surgery in cirrhotic patients. J Hepatol. 2012;57(4):874-884. doi:10.1016/j.jhep.2012.03.037

8. Nicoll A. Surgical risk in patients with cirrhosis J Gastroenterol Hepatol. 2012;27(10):1569-1575. doi:10.1111/j.1440-1746.2012.07205.x

9. Nikfarjam M, Shereef S, Kimchi ET, et al. Survival outcomes of patients with colorectal liver metastases following hepatic resection or ablation in the era of effective chemotherapy. Ann Surg Oncol. 2009;16(7):1860-1867. doi:10.1245/s10434-0080225-3

10. Pathak S, Tang JM, Terlizzo M, Poston GJ, Malik HZ. Hepatic steatosis, body mass index and long term outcome in patients undergoing hepatectomy for colorectal liver metastases. Eur J Surg Oncol. 2010;36(1):52-57. doi:10.1016/j.ejso.2009.09.004

11. Simonsen C, de Heer P, Bjerre ED, et al. Sarcopenia and postoperative complication risk in gastrointestinal surgical oncology: a meta-analysis. 
Ann Surg. 2018;268(1):58-69. doi:10.1097/SLA. 0000000000002679

12. Martin L, Birdsell L, Macdonald N, et al. Cancer cachexia in the age of obesity: skeletal muscle depletion is a powerful prognostic factor, independent of body mass index. J Clin Oncol. 2013 31(12):1539-1547. doi:10.1200/JCO.2012.45.2722

13. Zhang G, Meng S, Li R, Ye J, Zhao L. Clinical significance of sarcopenia in the treatment of patients with primary hepatic malignancies, a systematic review and meta-analysis. Oncotarget 2017;8(60):102474-102485. doi:10.18632/ oncotarget.19687

14. Lodewick TM, van Nijnatten TJ, van Dam RM, et al. Are sarcopenia, obesity and sarcopenic obesity predictive of outcome in patients with colorectal liver metastases? HPB (Oxford). 2015;17 (5):438-446. doi:10.1111/hpb.12373

15. Peng PD, van Vledder MG, Tsai $S$, et al. Sarcopenia negatively impacts short-term outcomes in patients undergoing hepatic resection for colorectal liver metastasis. HPB (Oxford). 2011;13 (7):439-446. doi:10.1111/j.1477-2574.2011.00301.x

16. Nam NH, Kaido T, Uemoto $\mathrm{S}$. Assessment and significance of sarcopenia in liver transplantation. Clin Transplant. 2019;33(12). doi:10.1111/ctr.13741

17. Coelen RJ, van Gulik TM. Preoperative sarcopenia negatively impacts postoperative outcomes following major hepatectomy with extrahepatic bile duct resection. World J Surg. 2015 39(9):2368-2369 doi:10.1007/s00268-015-3053-1

18. Pinto Dos Santos $D$, Kloeckner R, Koch $S$, et al. Sarcopenia as prognostic factor for survival after orthotopic liver transplantation. Eur J Gastroenterol Hepatol. 2020;32(5):626-634. doi:10.1097/MEG. 0000000000001552

19. Harimoto N, Hoshino H, Muranushi R, et al. Skeletal muscle volume and intramuscular adipose tissue are prognostic predictors of postoperative complications after hepatic resection. Anticancer Res. 2018;38(8):4933-4939. doi:10.21873/anticanres. 12810

20. Kobayashi A, Kaido T, Hamaguchi Y, et al Impact of sarcopenic obesity on outcomes in patients undergoing hepatectomy for hepatocellular carcinoma. Ann Surg. 2019;269(5): 924-931. doi:10.1097/SLA.0000000000002555

21. Chumlea WC, Roche AF. Ultrasonic and skinfold caliper measures of subcutaneous adipose tissue thickness in elderly men and women. Am J Phys Anthropol. 1986;71(3):351-357. doi:10.1002/ajpa. 1330710310

22. Santos LAA, Lima TB, letsugu MDV, Nunes HRC, Qi X, Romeiro FG. Anthropometric measures associated with sarcopenia in outpatients with live cirrhosis. Nutr Diet. 2019;76(5):613-619. doi:10.1111/ 1747-0080.12523

23. Abe T, Counts BR, Barnett BE, Dankel SJ, Lee K, Loenneke JP. Associations between handgrip strength and ultrasound-measured muscle thickness of the hand and forearm in young men and women. Ultrasound Med Biol. 2015;41(8):21252130. doi:10.1016/j.ultrasmedbio.2015.04.004
24. Lauretani $F$, Russo CR, Bandinelli $S$, et al. Age-associated changes in skeletal muscles and their effect on mobility: an operational diagnosis of sarcopenia. J Appl Physiol (1985). 2003;95(5): 1851-1860. doi:10.1152/japplphysiol.00246.2003

25. Kulkarni SS, Chen H, Josbeno DA, et al. Gait speed and grip strength are associated with dropping out of the liver transplant waiting list. Transplant Proc. 2019;51(3):794-797. doi:10.1016/j. transproceed.2019.01.030

26. Cruz-Jentoft AJ, Bahat G, Bauer J, et al; Writing Group for the European Working Group on Sarcopenia in Older People 2 (EWGSOP2), and the Extended Group for EWGSOP2. Sarcopenia: revised European consensus on definition and diagnosis. Age Ageing. 2019;48(4):601. doi:10.1093/ageing/ afz046

27. Santambrogio R, Kluger MD, Costa $M$, et al. Hepatic resection for hepatocellular carcinoma in patients with Child-Pugh's A cirrhosis: is clinical evidence of portal hypertension a contraindication? HPB (Oxford). 2013;15(1):78-84. doi:10.1111/j.14772574.2012.00594.x

28. Bosch J, García-Pagán JC. Complications of cirrhosis, I: portal hypertension. J Hepatol. 2000;32 (1)(suppl):141-156. doi:10.1016/S0168-8278(00) 80422-5

29. Charlson ME, Pompei P, Ales KL, MacKenzie CR. A new method of classifying prognostic comorbidity in longitudinal studies: development and validation. J Chronic Dis. 1987;40(5):373-383. doi:10.1016/0021-9681(87)90171-8

30. Dindo D, Demartines N, Clavien PA. Classification of surgical complications: a new proposal with evaluation in a cohort of 6336 patients and results of a survey. Ann Surg. 2004; 240(2):205-213. doi:10.1097/01.sla.0000133083. 54934.ae

31. Slankamenac K, Graf R, Barkun J, Puhan MA, Clavien PA. The comprehensive complication index: a novel continuous scale to measure surgical morbidity. Ann Surg. 2013;258(1):1-7. doi:10.1097/ SLA.0b013e318296c732

32. Ishizawa T, Hasegawa K, Kokudo N, et al. Risk factors and management of ascites after liver resection to treat hepatocellular carcinoma. Arch Surg. 2009;144(1):46-51. doi:10.1001/archsurg.2008.

33. Rahbari NN, Garden OJ, Padbury R, et al. Posthepatectomy liver failure: a definition and grading by the International Study Group of Liver Surgery (ISGLS). Surgery. 2011;149(5):713-724. doi: 10.1016/j.surg.2010.10.00

34. Koch M, Garden OJ, Padbury R, et al. Bile leakage after hepatobiliary and pancreatic surgery: a definition and grading of severity by the International Study Group of Liver Surgery. Surgery. 2011;149(5):680-688. doi:10.1016/j.surg.2010.12.002

35. World Medical Association. World Medical Association Declaration of Helsinki: ethical principles for medical research involving human subjects. JAMA. 2013;310(20):2191-2194. doi:10 1001/jama.2013.281053
36. Horowitz M, Neeman E, Sharon E, Ben-Eliyahu $\mathrm{S}$. Exploiting the critical perioperative period to improve long-term cancer outcomes. Nat Rev Clin Oncol. 2015;12(4):213-226. doi:10.1038/nrclinonc. 2014.224

37. Morley JE, Argiles JM, Evans WJ, et al; Society for Sarcopenia, Cachexia, and Wasting Disease. Nutritional recommendations for the management of sarcopenia. J Am Med Dir Assoc. 2010;11(6):391396. doi:10.1016/j.jamda.2010.04.014

38. Eriksson S, Nilsson JH, Strandberg Holka P, Eberhard J, Keussen I, Sturesson C. The impact of neoadjuvant chemotherapy on skeletal muscle depletion and preoperative sarcopenia in patients with resectable colorectal liver metastases. HPB (Oxford). 2017;19(4):331-337. doi:10.1016/j.hpb.2016 11.009

39. Sultan $\mathrm{P}$, Hamilton MA, Ackland GL. Preoperative muscle weakness as defined by handgrip strength and postoperative outcomes: a systematic review. BMC Anesthesiol. 2012;12:1. doi:10.1186/1471-2253-12-1

40. Verweij NM, Schiphorst $A H$, Pronk $A$, van den Bos F, Hamaker ME. Physical performance measures for predicting outcome in cancer patients: a systematic review. Acta Oncol. 2016;55 (12):1386-1391. doi:10.1080/0284186X.2016.1219047

41. Leong DP, Teo KK, Rangarajan S, et al; Prospective Urban Rural Epidemiology (PURE) Study Investigators. Prognostic value of grip strength: findings from the Prospective Urban Rura Epidemiology (PURE) study. Lancet. 2015;386 (9990):266-273 doi:10.1016/S0140-6736(14) 62000-6

42. Prado CM, Lieffers JR, McCargar LJ, et al. Prevalence and clinical implications of sarcopenic obesity in patients with solid tumours of the respiratory and gastrointestinal tracts: a population-based study. Lancet Oncol. 2008;9(7): 629-635. doi:10.1016/S1470-2045(08)70153-0

43. Seabolt LA, Welch EB, Silver HJ. Imaging methods for analyzing body composition in human obesity and cardiometabolic disease. Ann N Y Acad Sci. 2015;1353:41-59. doi:10.1111/nyas.12842

44. Fukuda Y, Yamamoto K, Hirao M, et al. Sarcopenia is associated with severe postoperative complications in elderly gastric cancer patients undergoing gastrectomy. Gastric Cancer. 2016:19 (3):986-993. doi:10.1007/s10120-015-0546-4 45. Huang DD, Zhou CJ, Wang SL, et al. Impact of different sarcopenia stages on the postoperative outcomes after radical gastrectomy for gastric cancer. Surgery. 2017;161(3):680-693. doi:10.1016/j. surg.2016.08.030

46. Huang DD, Wang $S L$, Zhuang $C L$, et al. Sarcopenia, as defined by low muscle mass, strength and physical performance, predicts complications after surgery for colorectal cancer. Colorectal Dis. 2015;17(11):0256-0264. doi:10.1111/ codi.13067 